\title{
Empleabilidad e inserción profesional de la formación de posgrado en el Sistema Universitario Español (SUE)
}

\author{
Employability and professional insertion of postgraduate \\ training in the Spanish University System
}

María Ángeles Alegre-Sánchez

UNIVERSIDAD NACIONAL DE EDUCACIÓN A DISTANCIA (UNED)

\section{Gemma Fonrodona-Baldajos}

UNIVERSIDAD DE BARCELONA (UB)

Antoni Vallès-Segalés

UNIVERSIDAD DE BARCELONA (UB)

Yolanda Agudo-Arroyo • yagudo@poli.uned.es

UNIVERSIDAD NACIONAL DE EDUCACIÓN A DISTANCIA (UNED)

Enviado: $25 / 01 / 2020$

Aceptado: 20/05/2020

\section{Resumen}

El Espacio Europeo de Educación Superior establece una nueva estructura de títulos de grado y posgrado para permitir la compatibilidad y la comparabilidad, poniendo el acento en la movilidad, la empleabilidad y la garantía de la calidad. Este trabajo tiene como objetivo analizar las diferencias en la inserción laboral entre los egresados y egresadas de máster y doctorado y al año y a los cuatro años de la finalización de los estudios, a partir de los datos de inserción laboral del informe de Datos y Cifras del Sistema Universitario Español 2015-16 (MECD, 2016) y desde un enfoque descriptivo comparativo y bivariante inferencial. Los resultados indican que España es el segundo país con una tasa de desempleo superior en los egresados de máster y presenta la tasa de desempleo más elevada de los egresados de doctorado de los países analizados. No se observan diferencias de género en cuanto a la tasa de afiliación, incluso siendo en muchos casos más favorable para las mujeres, sin embargo, a medida que se analizan indicadores más relacionados con la calidad del empleo, como el trabajo acorde y la base de cotización, se va incrementando la brecha de género en detrimento de las mujeres. Si bien, multitud de factores pueden dar lugar a estos resultados, resulta obvio que sigue siendo un reto impulsar políticas públicas para favorecer la igualdad de oportunidades de los egresados y las egresadas de la formación de posgrado.

Palabras clave: empleabilidad; inserción profesional; tasa de afiliación; trabajo acorde; base de cotización.

\section{Abstract}

The European Higher Education Area sets up a new structure of undergraduate and postgraduate degrees to enable compatibility and comparability, with an emphasis on mobility, employability and quality assurance. The aim of this work is to analyze the gender differences in the labor insertion of graduates of master's and doctoral degrees and one year and four years after completion of studies based on Spanish University System Data and Figures Report (MECD, 2016) and from a descriptive comparative and bivariate inferential approach. The results indicate that Spain is the second country with a higher unemployment rate among graduate Masters and has the highest unemployment rate among $\mathrm{PhD}$ graduates in the countries analyzed. There are no gender differences in the affiliation rates, even being in many cases more favorable for women. However, the gender gap increases to the detriment of women in indicators more related to the employment quality, such as work according to the educational level and the contribution basis. Although many factors may give rise to these results, it is clear that it remains a challenge to promote public policies for equal opportunities for graduates of the postgraduate training.

Keywords: employability, professional insertion; affiliation rates, quality work, contribution bases. 


\section{INTRODUCCIÓN}

El Espacio Europeo de Educación Superior (EEES) establece una nueva estructura de títulos de grado y posgrado con el objetivo de permitir la compatibilidad y la comparabilidad, poniendo el acento en el desarrollo de la movilidad y la empleabilidad universitarias, así como en la garantía de la calidad. De acuerdo con la Declaración de la Sorbona (1998), el reconocimiento internacional reside en las facilidades de comprensión y la nueva organización en ciclos es fundamental a la hora de establecer comparaciones y equivalencias a nivel internacional. En los estudios de posgrado, habría una opción entre un máster más corto y un doctorado más largo, con posibilidades de pasar de uno a otro. En ambos títulos de posgrado, se hará hincapié en la investigación y el trabajo autónomo. Reafirmando los principios de la Declaración de la Sorbona, la Declaración de Bolonia (1999) establece como uno de sus objetivos esenciales la adopción de un sistema de enseñanzas universitarias basado en ciclos, con un sistema de títulos fácilmente comprensibles y comparables en aras de promover la empleabilidad y la competitividad del sistema de enseñanza superior europeo a escala internacional.

En este mismo sentido se pronuncian las sucesivas conferencias ministeriales de seguimiento del EEES. El establecimiento del sistema de créditos ECTS y del Suplemento Europeo del Título (SET) son mencionados en la conferencia de Praga (2001) como medidas para progresar en el atractivo de la educación superior y el acceso del estudiantado al mercado laboral. El comunicado de Londres (2007) pone de relieve el avance realizado en la implantación del sistema de estudios en ciclos, debiendo concentrarse los esfuerzos en eliminar las barreras al acceso y la progresión entre ciclos, en los resultados de aprendizaje y la carga del trabajo del estudiante, así como en la necesidad de mejorar la empleabilidad y la recogida de datos. La conferencia de Bucarest (2012) indica la necesidad de un mayor esfuerzo en la transición entre los tres ciclos, el uso de los ECTS, del Suplemento al Título, del aseguramiento de la calidad y la implementación de marcos de calificaciones, incluyendo la definición y evaluación de los resultados de aprendizaje, e incentivando que los programas de estudio reflejen las disciplinas emergentes con miras a potenciar la empleabilidad. Recientemente, la conferencia de París (2018) explicita de manera especial la necesidad de seguir implementado los compromisos clave de Bolonia, poniendo especial énfasis en el sistema de tres ciclos compatible con el marco general de calificaciones del EEES.

En España, la Ley Orgánica 6/2001, de Universidades, modificada por la Ley Orgánica 4/2007, establece la estructura de las enseñanzas universitarias en tres ciclos: Grado, Máster y Doctorado, teniendo como finalidad los másteres universitarios la adquisición por el estudiante de una formación avanzada, de carácter especializado o multidisciplinar, orientada a la especialización académica o profesional, o a promover la iniciación en tareas investigadoras, según el Real Decreto 1393/2007, modificado por los RD 861/2010 y 43/2015. Por su parte, de acuerdo con el RD 99/2011, por el que se regulan las enseñanzas oficiales de doctorado, éste ha de jugar un papel fundamental como intersección entre el EEES y el Espacio Europeo de Investigación (EEI), los dos pilares fundamentales de 
la sociedad basada en el conocimiento. El componente fundamental de la formación doctoral es el avance del conocimiento científico a través de la «investigación original». Además, se considera que en este tercer ciclo los participantes en programas de doctorado no son nada más estudiantes sino investigadores en formación. Con ello se enlaza en este momento del proceso de Bolonia la formación doctoral, la carrera investigadora y la transmisión del conocimiento a la sociedad. Así, los estudios de doctorado tienen como finalidad la adquisición de competencias y habilidades relacionadas con la investigación científica de calidad. La expedición del Suplemento Europeo al Título Universitario de Doctor, queda regulada por el Real Decreto 195/2016, de 13 de mayo, que establece los requisitos para la expedición del mismo. Es precisamente en este marco que nos ha interesado analizar los resultados de inserción laboral de la formación de posgrado aportados por el informe $D a$ tos y Cifras del Sistema Universitario Español 2015-16 (Ministerio de Educación, Cultura y Deporte -MECD—, 2016) desde un enfoque descriptivo comparativo y bivariante inferencial en los niveles de posgrado. En concreto, el objetivo de este trabajo es analizar las diferencias en la tasa de afiliación, el trabajo acorde y la base de cotización en los niveles de máster y doctorado entre los hombres y las mujeres y entre los años 2011 y 2014.

\section{LA EMPLEABILIDAD EN EL MARCO DEL ESPACIO EUROPEO DE EDUCACIÓN SUPERIOR (EEES)}

Como ha quedado de manifiesto, los diferentes Comunicados de las Conferencias de Ministros Responsables de Educación Superior establecen los objetivos y prioridades de la educación superior en Europa, señalando la empleabilidad en el marco del EEES como un aspecto de gran relevancia. En este sentido, podemos señalar como síntesis de sus prioridades en este ámbito:

- Incrementar la empleabilidad, instrumento para aprovechar plenamente las oportunidades del cambiante mercado laboral, el desarrollo personal y profesional, en cada uno de los tres ciclos y en el contexto de aprendizaje a lo largo de la vida.

- Desarrollar programas de estudio que incrementen el potencial de innovación, emprendimiento e investigación de los estudiantes, con un buen equilibrio entre los componentes teóricos y prácticos.

- Dotar a los alumnos de conocimientos, habilidades y competencias necesarios para responder a los retos del nuevo milenio y preparar al estudiante para el mercado laboral. Una atención especial requiere el fomento de las habilidades y competencias digitales, preparando a los estudiantes para actuar de forma creativa en un entorno digital. Y se pone de relieve la necesidad de combinar la formación de habilidades y competencias transversales y multidisciplinares con las específicas de la carrera elegida.

- Mejorar y fortalecer la cooperación entre empleadores, estudiantes e instituciones.

- Potenciar los servicios de orientación profesional y de empleo universitarios.

- Realizar el seguimiento del desarrollo de la carrera profesional de los graduados. 
Así, en el marco del EEES el concepto de empleabilidad debemos entenderlo desde un enfoque dinámico, proactivo, de proceso continuado en relación al desarrollo y carrera profesional. Según el informe español Objetivos educativos europeos y españoles. Estrategia de Educación y Formación 2020 (MECD, 2013) «la empleabilidad se define como la combinación de factores que permiten a los individuos progresar hacia el empleo o acceder a él, permanecer en un trabajo o progresar durante una carrera laboral; es un concepto complejo que incluye tanto las características, habilidades, actitudes y motivación individuales como factores externos que van más allá de las políticas educativas y de formación, tales como la regulación del mercado, la demografía, la estructura del marco económico o la situación económica general» (p.47). Si bien, «no debe olvidarse que bajo el concepto de empleabilidad de una persona no sólo se entiende la posesión de una serie de competencias requeridas para satisfacer las cambiantes demandas de los empleadores (mantener el empleo), sino también aquellas requeridas para inicialmente obtenerlo» (Rodríguez et al., 2010, p. 111).

En el marco del cambio de paradigma al proceso de enseñanza-aprendizaje centrado en el estudiante que supone el EEES, la adquisición de competencias deviene esencial, así lo pone de relieve el proyecto Tuning Educational Structures in Europe (González y Wagenaar, 2003), poniendo el acento no solamente en la importancia de la asunción de competencias específicas sino también en la relevancia del desarrollo de las competencias genéricas, más aún en un contexto de aprendizaje a lo largo de la vida. En este sentido, afirma «The Tuning Project deals with two types of competences: generic competences (instrumental, interpersonal and systemic) and subject-specific competences (including skills and knowledge)» (p. 28). Por su parte, Bravo Salinas (2007) señala que Tuning define las competencias como «una combinación dinámica de atributos, en relación a procedimientos, habilidades, actitudes y responsabilidades, que describen los encargados del aprendizaje de un programa educativo o lo que los alumnos son capaces de demostrar al final de un proceso educativo» (p. 13). Echeverría y Martínez Clares (2018) consideran que el nuevo escenario laboral requiere no solamente competencias de carácter técnico o metodológico sino sobretodo competencias participativas y personales, ampliamente generalizables y transferibles a diferentes contextos y actividades, jugando la educación un poderoso rol para la actualización y mejora de las competencias a lo largo de la vida. En el marco del Sistema Universitario Español (SUE) los referentes normativos en cuanto a las competencias que deben adquirir los estudiantes de posgrado las encontramos recogidas en los RD 1393/2007, RD 861/2010 y en el RD 1027/2011 del Marco Español de Cualificaciones para la Educación Superior (MECES), diseñado a partir del Marco Europeo Global de Cualificaciones (EQF).

El informe el Espacio Europeo de Educación Superior en 2015: Informe sobre la implantación del Proceso de Bolonia (Comisión Europea/EACEA/Eurydice, 2015) incide en el hecho de que los responsables políticos deben poner una especial atención en la empleabilidad de los titulados, dado además el contexto de crisis económica que lo hace todavía más necesario, y subraya que, si bien se tiene como una de las prioridades del EEES, no 
en todos los sistemas se desarrollan esfuerzos sistemáticos para fortalecer dicha empleabilidad, entre los que cita, el análisis de las previsiones del mercado laboral, la participación de las empresas, los incentivos para ofrecer contratos en prácticas en muchos programas de educación superior, la mejora de los servicios de orientación profesional, el seguimiento del rendimiento de las instituciones a través de sistemas de evaluación bien establecidos y el fomento de la movilidad de los estudiantes, sin detrimento de reconocer que la mayor parte de países han puesto en marcha políticas y mecanismos de seguimiento en este ámbito.

Así, en la línea de potenciar la empleabilidad y las competencias del estudiantado universitario deben seguir poniendo una especial atención las políticas educativas de educación superior y, sin duda, poner el acento en favorecer la reducción de desigualdades de género, ya que son muchos los informes que aportan datos empíricos en esta dirección. En este sentido, los últimos datos publicados por el Informe de Inserción Laboral de los Egresados Universitarios (Ministerio de Ciencia, Innovación y Universidades, 2019) ponen de relieve la existencia de diferencias de género en la inserción profesional del estudiantado titulado de grado y, basándose en estos datos, el informe Esenciales, n. 39 (BBVA-IVIE, 2019), muestra que, si bien las universitarias presentan tasas de rendimiento académico, tasas de idoneidad en la graduación y tasas de graduación superiores a los universitarios, sus tasas de afiliación a la Seguridad Social y sus bases de cotización son inferiores. Estos datos concuerdan con lo afirmado por el Informe Español. Panorama de la Educación (OCDE, 2018), que pone de relieve que la brecha de género en el sistema educativo opera a favor de las mujeres, pero lo hace a favor de los hombres en el mercado laboral.

Vista la relevancia de la empleabilidad en la estructura de enseñanzas en el EEES y, en concreto, en el nivel de posgrado y a la luz de las conclusiones sobre la brecha de género en los indicadores de inserción laboral de los y las graduadas universitarias de los informes revisados, nos planteamos las siguientes hipótesis:

- H1a: La tasa de afiliación es superior en los egresados de master y doctorado que en las egresadas tanto en 2011 como en 2014.

— H1b. La tasa de afiliación se incrementa entre el 2011 y 2014 tanto en los egresados como en las egresadas de master y doctorado.

- H2a. El trabajo acorde es superior en los egresados de master y doctorado que en las egresadas tanto en 2011 como en 2014.

- H2b. El trabajo acorde disminuye entre el 2011 y 2014 tanto en los egresados como en las egresadas de master y doctorado.

- H3a. La base de cotización es superior en los egresados de master y doctorado que en las egresadas tanto en 2011 como en 2014.

- H3b. La base de cotización se incrementa entre el 2011 y 2014 tanto en los egresados como en las egresadas de master y doctorado. 


\section{OBJETIVO}

El objetivo de este trabajo es analizar las diferencias en los indicadores de inserción laboral de la tasa de afiliación, el trabajo acorde y la base de cotización en los niveles de posgrado de mster y doctorado entre los hombres y las mujeres y entre los años 2011 y 2014.

\section{PROCEDIMIENTO}

\section{Datos}

Para este trabajo hemos utilizado los resultados de inserción laboral de la formación de posgrado aportados por el informe Datos y Cifras del Sistema Universitario Español 20152016 (MECD, 2016). Tal y como indica el Informe de Inserción laboral de los egresados universitarios. La perspectiva de la afiliación a la Seguridad Social (MECD, 2015), el informe tiene como objetivo diagnosticar desde la perspectiva de la afiliación a la Seguridad Social el proceso de inserción laboral de la cohorte de egresados y egresadas del curso académico 2009-10. En este sentido, la población contemplada para el caso de los estudios de posgrado es de 40.391 egresados de master y de 8.527 egresados de doctorado. Y realiza un análisis transversal de dicha cohorte poniendo en relación la información del Sistema Integrado de Información Universitaria (SIIU) con la de la Tesorería de la Seguridad Social, proporcionando información en base a los indicadores de tasa de empleo y desempleo, tasa de afiliación (altas en la Seguridad Social en un momento determinado), porcentaje de egresados de posgrado en el régimen especial de autónomos, porcentaje de egresados de posgrado con contrato indefinido, porcentaje de egresados de posgrado que realizan un trabajo acorde con su nivel formativo y base de cotización anual estudios de posgrado.

\section{Variables}

Para analizar las diferencias en los indicadores de la tasa de afiliación, el trabajo acorde y la base de cotización entre los egresados y las egresadas de master y doctorado y entre los años 2011 y 2014, tomamos como variables dependientes la tasa de afiliación, el trabajo acorde y la base de cotización y como variables independientes el sexo y los años 2011 y 2014.

\section{Técnicas de análisis cuantitativo}

Para validar las hipótesis H1a y H1b relativas a la tasa de afiliación y las hipótesis H2a y $\mathrm{H} 2 \mathrm{~b}$ relativas al trabajo acorde, se ha procedido en primer lugar a calcular las tasas medias de afiliación y de trabajo acorde en máster y doctorado de los hombres y de las mujeres y en los años 2011 y 2014, realizando posteriormente un contraste de hipótesis para 
dos proporciones mediante la prueba Z. Para validar las hipótesis H3a y H3b relativas a la base de cotización, se calculan las bases medias de cotización en máster y doctorado de los hombres y de las mujeres y en los años 2011 y 2014, y se realiza un contraste de hipótesis para dos medias de muestras independientes para analizar las diferencias entre hombres y mujeres y un contraste de hipótesis para dos medias de muestras relacionadas para comparar las diferencias en los dos momentos analizados: año 2011 y año 2014. Para el análisis de los datos se ha utilizado el programa estadístico Minitab versión 17.

\section{RESULTADOS DE LA FORMACIÓN DE POSGRADO EN EL SISTEMA UNIVERSITARIO ESPAÑOL (SUE)}

De acuerdo con nuestro objetivo, los resultados que aquí se presentan se centrarán en analizar las diferencias de los indicadores correspondientes a la tasa de afiliación, el trabajo acorde y la base de cotización en los niveles de la formación de posgrado, máster y doctorado, entre hombres y mujeres y en los años 2011 y 2014. Si bien, nos permitimos hacer una referencia introductoria a los datos de desempleo en la población de posgrado presentados por el citado informe en aras de llamar la atención sobre las diferencias con los países de la OCDE y UE.

Así, en cuanto a la tasa de desempleo de los egresados de master y doctorado en el año 2014 (Gráfico 1), los resultados indican que España es el segundo país con una tasa de desempleo superior en los egresados de máster y presenta la tasa de desempleo más elevada de los egresados de doctorado de los países analizados.

La tasa de afiliación mide el porcentaje de egresados universitarios que están en alta en la Seguridad Social en una fecha determinada de los años naturales siguientes a la finalización de su titulación universitaria (MECD, 2015). En el caso del informe se analiza esta tasa en dos momentos concretos 2011 y 2014.

Los resultados indican que en el caso de los egresados de máster la tasa de afiliación entre 2011 y 2014 se incrementa en 7,4 puntos porcentuales, mientras que en el caso de los egresados de doctorado experimenta una disminución de 1,6 puntos porcentuales. Por ramas de enseñanza en el caso de los estudios de máster el incremento se produce en todas ellas, siendo más destacado en el caso de Ciencias Sociales y Jurídicas y Artes y Humanidades, mientras que en los estudios de doctorado se produce una disminución en todas las ramas de enseñanza, siendo más acusada en la rama técnica de Ingeniería y Arquitectura y en la de Ciencias.

En el caso de las ramas de Ingeniería y Arquitectura y Artes y Humanidades la tasa de afiliación en el 2014 es superior en máster que en doctorado y en la rama de Ciencias el máster posee mayor afiliación que el doctorado tanto en 2011 como en 2014. 
INGURUAK [68] | 2020 | 1-20

Gráfico 1. Tasa de desempleo (\%) de la población de 25 a 64 años de los egresados de Máster y Doctorado, correspondiente al año 2014

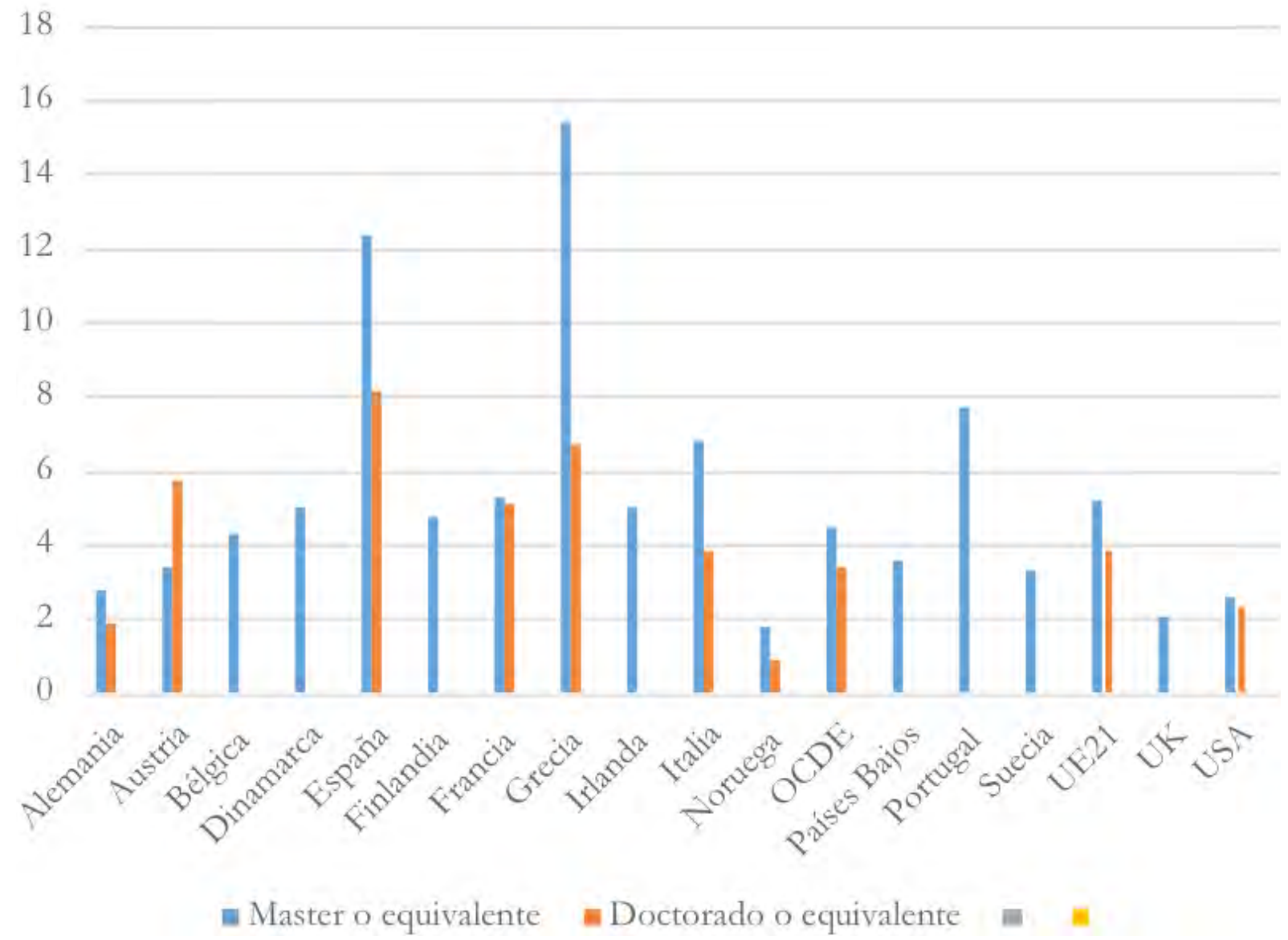

Fuente: elaboración propia. Datos MECD.

Gráfico 2. Tasa de afiliación de los egresados de Máster y Doctorado en 2011 y 2014 por rama de enseñanza (\%). Cohorte de egresados en el curso 2009-10

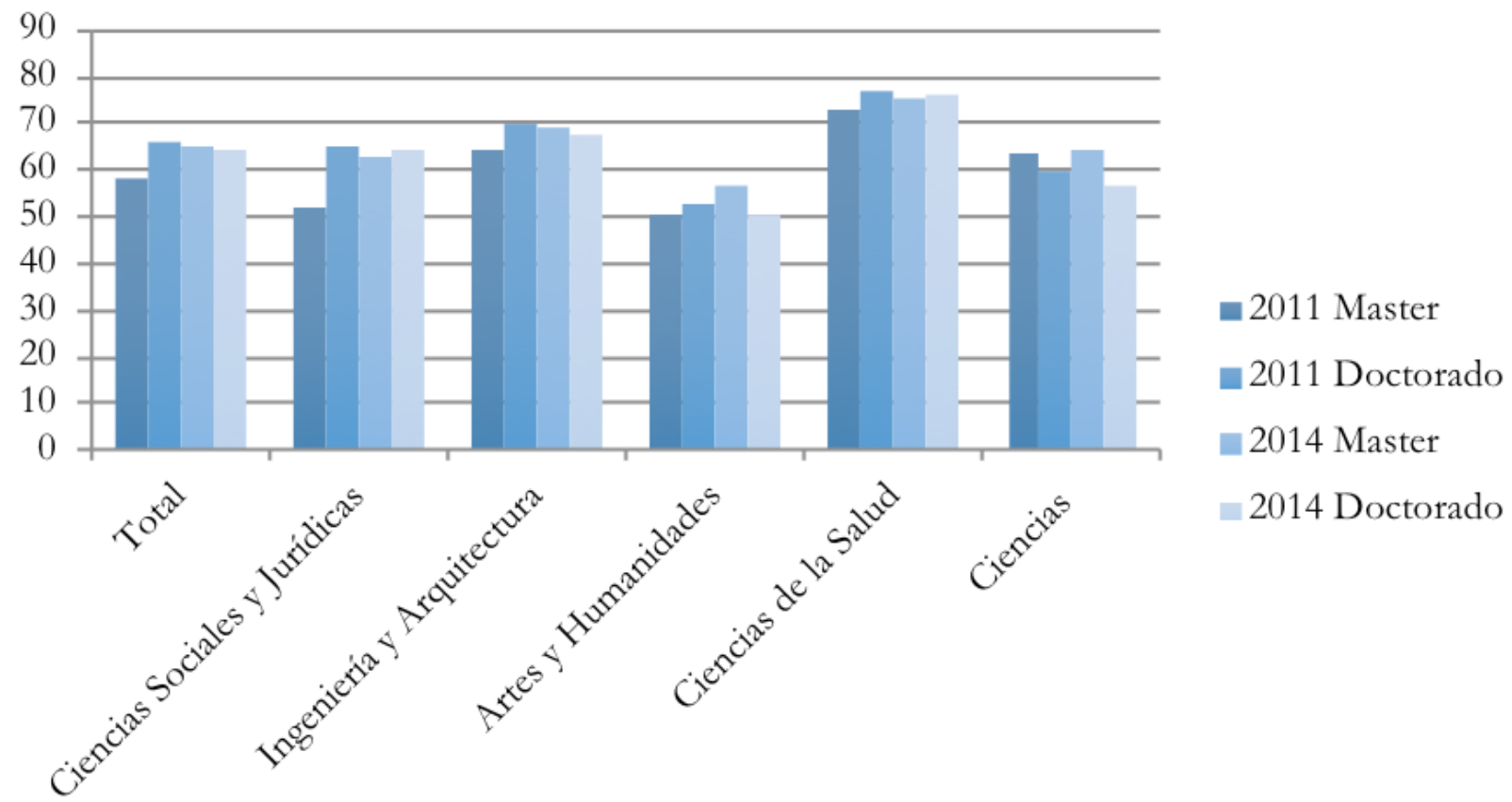

Fuente: elaboración propia. Datos MECD. 
En cuanto a la tasa de afiliación por ámbitos de estudios a los cuatro años de haber finalizado los estudios y en función de los datos analizados, se observa que la mayor tasa de afiliación en el caso del máster se da en el ámbito de estudios de Servicios de Transporte $(81,8 \%)$ y la menor en Humanidades (51,5\%), mientras que en el caso del doctorado el de mayor afiliación es Servicios Personales (85,7\%) y el de menor de nuevo en Humanidades (48,5\%).

Gráfico 3. Tasa de afiliación de los egresados de Máster y Doctorado en 2014 por ámbitos de estudio (\%). Cohorte de egresados en el curso 2009-10

Administración y Negocios

Agricultura, ganadería y pesca

Arquitectura y construcción

Artes

Ciencias

Ciencias de la vida

Ciencias físicas, químicas y geológicas

Ciencias sociales y del comportamiento

Derecho

Formación de personal docente

Humanidades

Ind. Manufacturera y producción

Informática

Ingeniería y profesiones afines

Matemáticas y estadística

Periodismo e información

Protección del medio ambiente

Salud

Servicios de seguridad

Servicios de transporte

Servicios personales

Servicos Sociales

Veterinaria



Fuente: elaboración propia. Datos MECD.

Analizada la tasa de afiliación por rama de enseñanza y sexo en el año 2011, los resultados indican que en el caso de los egresados de máster la tasa de afiliación es superior para los hombres que para las mujeres en Ciencias Sociales y Jurídicas e Ingeniería y Arquitec- 
tura, siendo ligeramente superior para las mujeres en el caso de las ramas de enseñanza de Ciencias de la Salud, Artes y Humanidades y Ciencias.

Respecto a la tasa de afiliación de los egresados de doctorado en 2011, se observa que las mujeres tienen una tasa de afiliación superior en todas las ramas de conocimiento, a excepción del área de Ciencias de la Salud que presenta una diferencia poco significativa, dándose la diferencia más significativa a favor de las mujeres en la rama de enseñanza de Ciencias Sociales y Jurídicas, seguida de la de Ciencias.

Gráfico 4. Tasa de afiliación de los egresados de Máster y Doctorado en 2011 por rama de enseñanza y sexo (\%). Cohorte de egresados en el curso 2009-2010

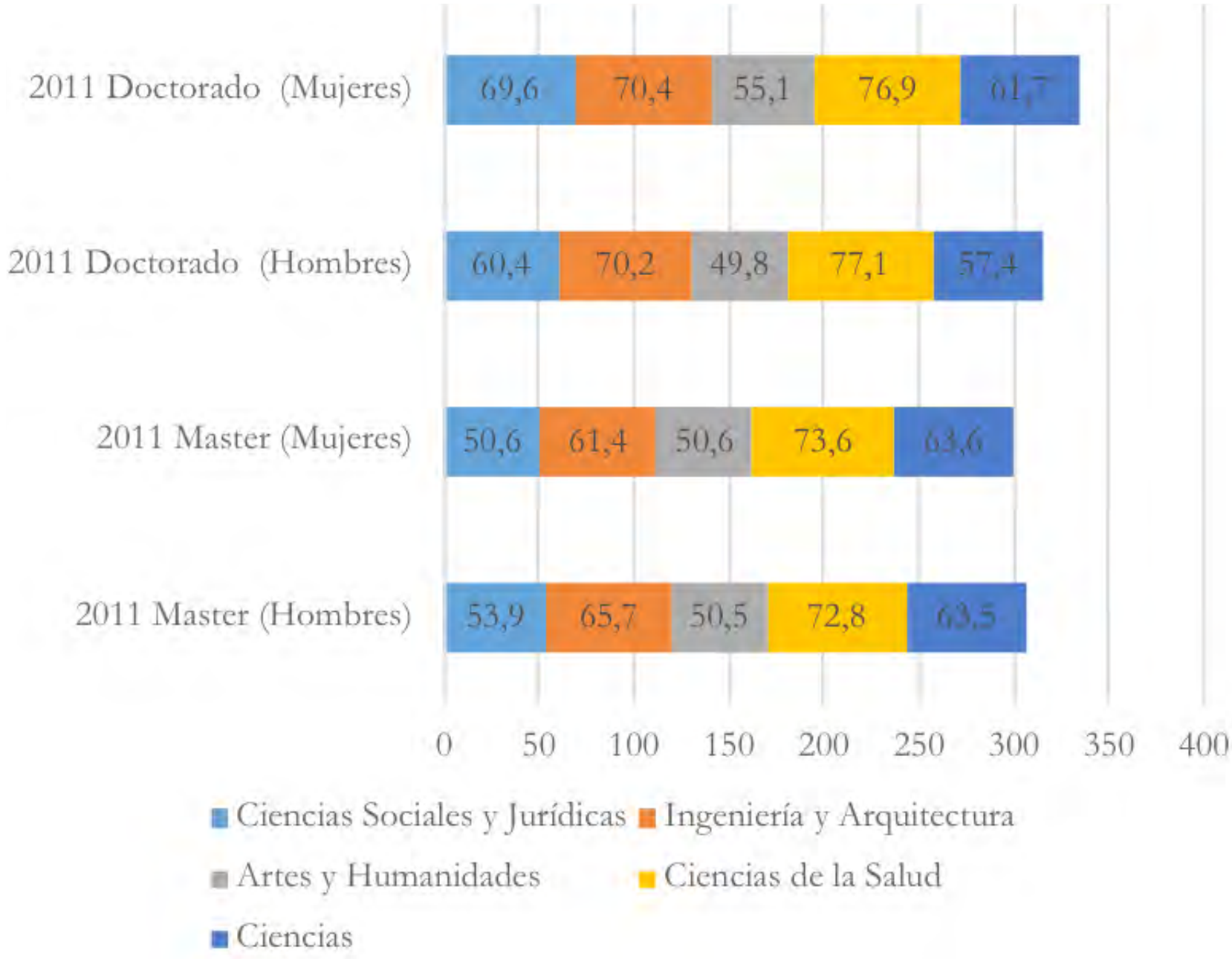

Fuente: elaboración propia. Datos MECD.

Los resultados en cuanto a la tasa de afiliación por rama de enseñanza y sexo en el año 2014 indican que, en el caso de máster, al igual que en el año 2011, la tasa es superior para los hombres en las ramas de enseñanza de Ciencias Sociales y Jurídicas e Ingeniería y Arquitectura, mientras que lo es para las mujeres en Artes y Humanidades, Ciencias de la Salud y Ciencias. En el caso de los estudios de doctorado las tasas de afiliación son superiores para las mujeres en todas las ramas de enseñanza, dándose las mayores diferencias a fa- 
vor de las mujeres en la rama de Ciencias Sociales y Jurídicas, seguida de las ramas de Artes y Humanidades y de Ciencias.

Gráfico 5. Tasa de afiliación de los egresados de Máster y Doctorado en 2014 por rama de enseñanza y sexo (\%). Cohorte de egresados en el curso 2009-10

\section{Doctorado (Mujeres)}

2014 Doctorado (Hombres)

2014 Master (Mujeres)

2014 Master (Hombres



- Ciencias Sociales y Jurídicas Ingeniería y Arquitectura

Artes y Humanidades Ciencias de la Salud

= Ciencias

Fuente: elaboración propia. Datos MECD.

Los resultados indican que hay diferencias significativas en la tasa de afiliación de máster en el año 2011 entre los hombres y las mujeres a favor de los hombres, mientras que hay diferencias significativas en la tasa de afiliación de doctorado entre los hombres y las mujeres tanto en 2011 como en 2014 a favor de las mujeres (Tabla 1).

Tabla 1. Tasa de afiliación de Máster y Doctorado. Sexo

\begin{tabular}{|l|c|c|c|c|c|c|c|c|}
\hline \multirow{2}{*}{} & \multicolumn{4}{|c|}{ Tasa de afiliación 2011 } & \multicolumn{3}{c|}{ Tasa de afiliación 2014 } \\
\cline { 2 - 9 } & \multicolumn{2}{|c|}{ Máster } & \multicolumn{2}{c|}{ Doctorado } & \multicolumn{2}{c|}{ Máster } & \multicolumn{2}{c|}{ Doctorado } \\
\cline { 2 - 9 } & Hombres & Mujeres & Hombres & Mujeres & Hombres & Mujeres & Hombres & Mujeres \\
\hline Media & 61,28 & 59,96 & 62,98 & 66,74 & 65,88 & 65,46 & 60,28 & 66,16 \\
\hline$\rho$-valor & \multicolumn{2}{|c|}{$\mathbf{0 , 0 0 3}$} & \multicolumn{2}{c|}{0,000} & \multicolumn{2}{c|}{0,383} & \multicolumn{2}{c}{$\mathbf{0 , 0 0 0}$} \\
\hline
\end{tabular}

Fuente: elaboración propia. Datos MECD. 
La tasa de afiliación de máster se incrementa de manera significativa entre 2011 y 2014, tano en los hombres como en las mujeres, mientras que la tasa de afiliación de doctorado experimenta un descenso significativo entre 2011 y 2014 en el caso de los hombres (Tabla 2).

Tabla 2. Tasa de afiliación de Máster y Doctorado. 2011 y 2014

\begin{tabular}{|l|c|c|c|c|c|c|c|c|}
\hline \multirow{2}{*}{} & \multicolumn{3}{|c|}{ Tasa de afiliación Máster } & \multicolumn{3}{c|}{ Tasa de afiliación Doctorado } \\
\cline { 2 - 9 } & \multicolumn{2}{|c|}{ Mujeres } & \multicolumn{2}{c|}{ Hombres } & \multicolumn{2}{c|}{ Mujeres } & \multicolumn{2}{c|}{ Hombres } \\
\cline { 2 - 9 } & $\mathbf{2 0 1 1}$ & $\mathbf{2 0 1 4}$ & 2011 & $\mathbf{2 0 1 4}$ & 2011 & 2014 & 2011 & 2014 \\
\hline Media & 59,96 & 65,46 & 61,28 & 65,88 & 66,74 & 66,16 & 62,98 & 60,28 \\
\hline$\rho$-valor & \multicolumn{2}{|c|}{0,000} & \multicolumn{2}{c|}{0,000} & \multicolumn{2}{c|}{0,561} & \multicolumn{2}{c|}{$\mathbf{0 , 0 0 6}$} \\
\hline
\end{tabular}

Fuente: elaboración propia. Datos MECD.

En relación al trabajo acorde al nivel formativo, los resultados indican que para el año 2011 los hombres tienen porcentajes superiores de trabajo acorde que las mujeres en todas las ramas de enseñanza en el caso del máster, mientras que para el caso del doctorado los porcentajes superiores de trabajo acorde de los hombres respecto de las mujeres se dan en las ramas de enseñanza de Ingeniería y Arquitectura, Ciencias de la Salud y Ciencias. A los cuatro años de finalizar los estudios, en el 2014, los hombres siguen teniendo mayores porcentajes de trabajo acorde que las mujeres en todas las ramas de enseñanza en el caso de los estudios de máster, mientras que esta misma situación se produce, al igual que en el año 2011, en el doctorado solo para las ramas de enseñanza de Ingeniería y Arquitectura, Ciencias de la Salud y Ciencias.

Gráfico 6. Trabajo acorde Máster y Doctorado 2011 y 2014 por rama de enseñanza y sexo. (\%). Cohorte de egresados en el curso 2009-10

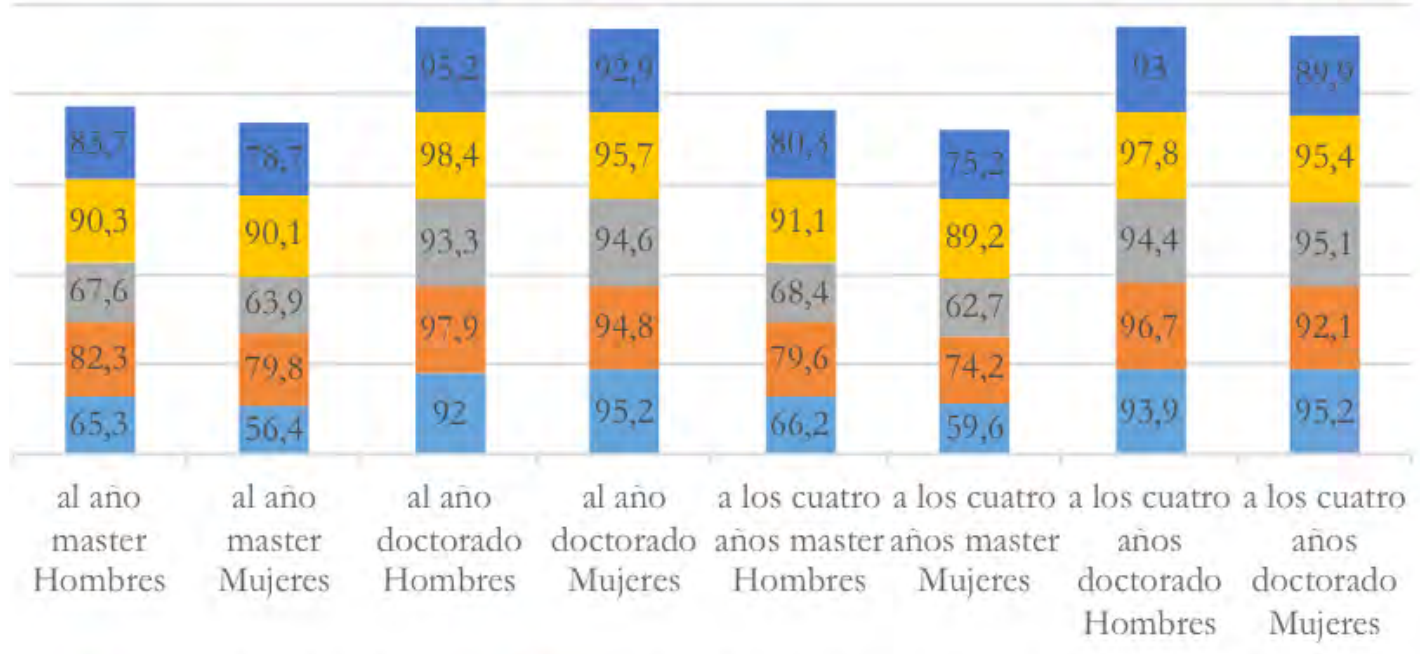

a Ciencias Sociales y Jurídicas Ingeniería y Arquitectura

- Ciencias de la Salud a Ciencias

Fuente: elaboración propia. Datos MECD. 
En el indicador de trabajo acorde en los estudios de máster hay diferencias significativas entre los hombres y las mujeres tanto en el 2011 como en el 2014, a favor de los hombres. Se observan también diferencias significativas en doctorado entre ambos sexos en el 2014, también a favor de los hombres (Tabla 3).

Tabla 3. Trabajo acorde de Máster y Doctorado. Sexo

\begin{tabular}{|l|c|c|c|c|c|c|c|c|}
\hline \multirow{2}{*}{} & \multicolumn{4}{|c|}{ Trabajo acorde 2011 } & \multicolumn{3}{c|}{ Trabajo acorde 2014 } \\
\cline { 2 - 9 } & \multicolumn{2}{|c|}{ Máster } & \multicolumn{2}{c|}{ Doctorado } & \multicolumn{2}{c|}{ Máster } & \multicolumn{2}{c|}{ Doctorado } \\
\cline { 2 - 9 } & Hombres & Mujeres & Hombres & Mujeres & Hombres & Mujeres & Hombres & Mujeres \\
\hline Media & 77,84 & 73,78 & 95,36 & 94,64 & 77,12 & 72,18 & 95,16 & 93,54 \\
\hline$\rho$-valor & \multicolumn{2}{|c|}{$\mathbf{0 , 0 0 0}$} & \multicolumn{2}{c|}{0,069} & \multicolumn{2}{c|}{0,000} & \multicolumn{2}{c|}{$\mathbf{0 , 0 0 1}$} \\
\hline
\end{tabular}

Fuente: elaboración propia. Datos MECD.

Entre los años 2011 y 2014 se produce un descenso significativo del indicador de trabajo acorde al nivel formativo en las mujeres tanto en los estudios de máster como en doctorado (Tabla 4).

Tabla 4. Trabajo acorde de Máster y Doctorado. 2011 y 2014

\begin{tabular}{|l|c|c|c|c|c|c|c|c|}
\hline \multirow{2}{*}{} & \multicolumn{4}{|c|}{ Trabajo acorde Máster } & \multicolumn{3}{c|}{ Trabajo acorde Doctorado } \\
\cline { 2 - 9 } & \multicolumn{2}{|c|}{ Mujeres } & \multicolumn{2}{c|}{ Hombres } & \multicolumn{2}{c|}{ Mujeres } & \multicolumn{2}{c|}{ Hombres } \\
\cline { 2 - 9 } & $\mathbf{2 0 1 1}$ & $\mathbf{2 0 1 4}$ & $\mathbf{2 0 1 1}$ & $\mathbf{2 0 1 4}$ & $\mathbf{2 0 1 1}$ & $\mathbf{2 0 1 4}$ & $\mathbf{2 0 1 1}$ & $\mathbf{2 0 1 4}$ \\
\hline Media & 73,78 & 72,18 & 77,84 & 77,12 & 94,64 & 93,54 & 95,36 & 95,16 \\
\hline$\rho$-valor & \multicolumn{2}{|c|}{$\mathbf{0 , 0 0 0}$} & \multicolumn{2}{c|}{0,051} & \multicolumn{2}{c|}{$\mathbf{0 , 0 1 3}$} & \multicolumn{2}{c|}{$\mathbf{0 , 6 7 6}$} \\
\hline
\end{tabular}

Fuente: elaboración propia. Datos MECD.

Las bases de cotización tanto para al año de finalizar los estudios, 2011, como a los cuatro años, 2014, y tanto para máster como doctorado son superiores para los hombres que para las mujeres en todas las ramas de enseñanza.

En el caso del máster la diferencia total en el año 2011 es de $2.339 €$ y en 2014 de $3.474 €$, por tanto, se produce un aumento en la diferencia de $1.135 €$ entre los años 2011 y 2014 a favor de los hombres. La mayor diferencia en 2011 se da en los estudios de Ciencias Sociales y Jurídicas (3.620€) y Ciencias (3.219€), seguido por este orden de Ingeniería y Arquitectura (2.422€), Ciencias de la Salud (2.039€) y Artes y Humanidades (1.627€), asimismo en 2014 las ramas de enseñanza con mayores diferencias siguen siendo Ciencias Sociales y Jurídicas (4.373€) y Ciencias (3.339€), seguidas de Ingeniería y Arquitectura (2.820€), Ciencias de la Salud (2652€) y Artes y Humanidades (2.532€). Así, se observa un incremento significativo en la diferencia en las bases de cotización entre hombres y mujeres entre 2011 y 2014. 
En el caso de doctorado la diferencia entre las bases de cotización de los hombres y de las mujeres en 2011 es de $1446 €$ y en 2014 de 1.567€, por lo que, al igual que en el caso del máster, se da un incremento de la diferencia, pero en este caso mucho menos acusada, siendo de $121 €$ a favor de los hombres. Por ramas de enseñanza en el año 2011 la mayor diferencia se da en las ramas de Ciencias de la Salud (2.580€) e Ingeniería y Arquitectura (2.164€), seguidas de Ciencias Sociales y Jurídicas (1.704€), Artes y Humanidades (1.677€) y Ciencias (286€), mientras que en el año 2014 la mayor diferencia se sigue dando en Ciencias de la Salud (2.716€) e Ingeniería y Arquitectura (1828€), seguidas de Ciencias (1.668€), Artes y Humanidades (1.328€) y Ciencias Sociales y Jurídicas (654€). En el caso de doctorado se produce un incremento de las diferencias de las bases de cotización entre los años 2011 y 2014 entre hombres y mujeres en las ramas de enseñanza de Ciencias de la Salud y Ciencias, en este último caso más significativo, puesto que supone un aumento de la diferencia de $1.382 €$ a favor de los hombres, y, a su vez, se da un descenso de la diferencia en las ramas de enseñanza de Ingeniería y Arquitectura, Artes y Humanidades y Ciencias Sociales y Jurídicas, siendo el descenso de la diferencia en esta última rama de enseñanza el más significativo alcanzando los $1.050 €$.

Gráfico 7. Bases de cotización (en €) en 2011 y 2014 por rama de enseñanza y sexo

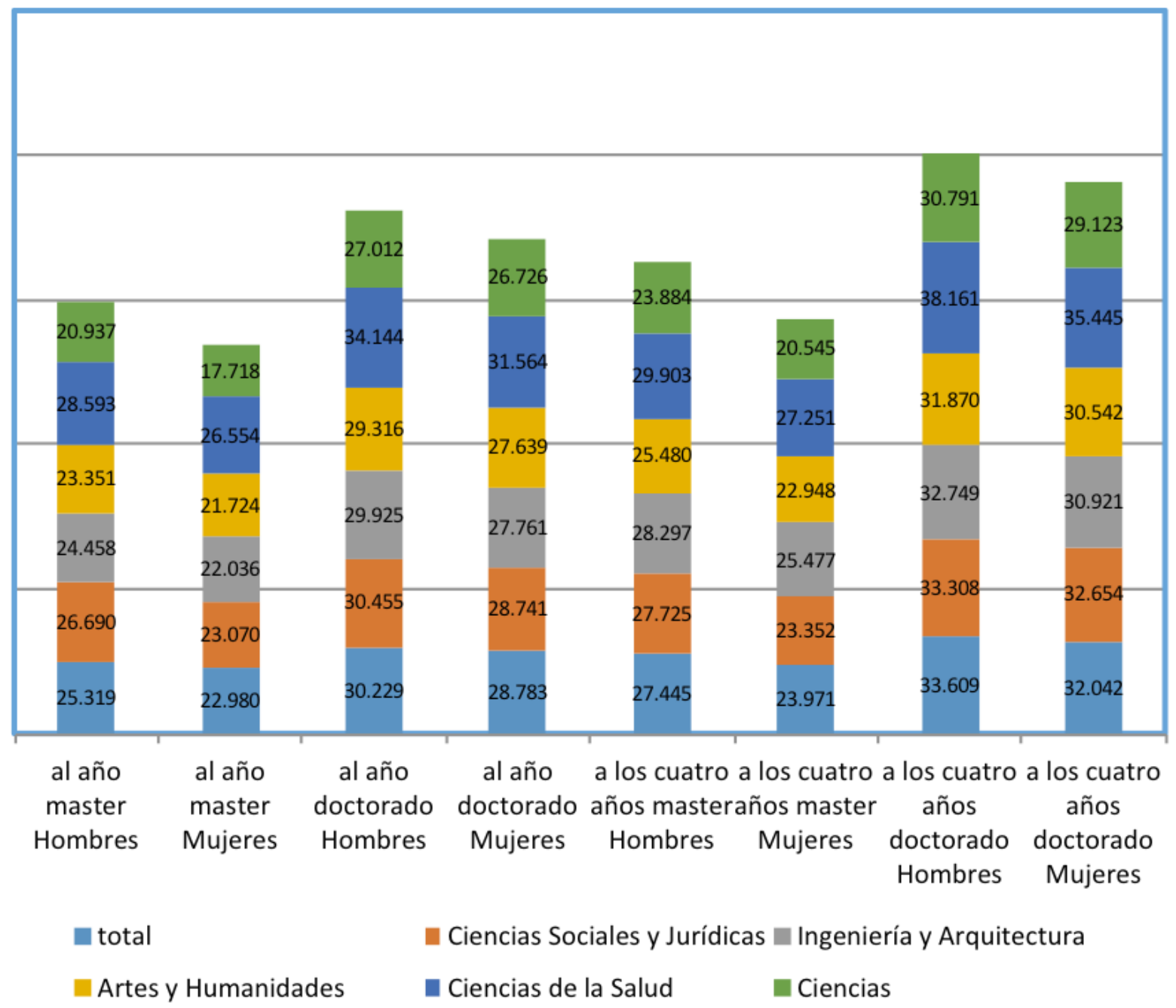

Fuente: elaboración propia. Datos del MECD. 
Los resultados indican que las bases de cotización son significativamente superiores para los hombres que para las mujeres tanto en máster como en doctorado y tanto en el año 2011 como en el 2014 (Tabla 5).

Tabla 5. Bases de cotización de Master y Doctorado. Sexo

\begin{tabular}{|c|c|c|c|c|c|c|c|c|}
\hline & \multicolumn{4}{|c|}{ Bases de cotización 2011} & \multicolumn{4}{|c|}{ Bases de cotización 2014} \\
\hline & \multicolumn{2}{|c|}{ Máster } & \multicolumn{2}{|c|}{ Doctorado } & \multicolumn{2}{|c|}{ Máster } & \multicolumn{2}{|c|}{ Doctorado } \\
\hline & Hombres & Mujeres & Hombres & Mujeres & Hombres & Mujeres & Hombres & Mujeres \\
\hline Media & 24806 & 22220 & 30171 & 28486 & 27058 & 23915 & 33376 & 31737 \\
\hline$\rho$-valor & \multicolumn{2}{|c|}{0,004} & \multicolumn{2}{|c|}{0,017} & \multicolumn{2}{|c|}{0,000} & \multicolumn{2}{|c|}{0,002} \\
\hline
\end{tabular}

Fuente: elaboración propia. Datos MECD.

El incremento de las bases de cotización es significativo entre el 2011 y 2014 tanto en hombres como mujeres y como en máster y doctorado (Tabla 6).

Tabla 6. Bases de cotización de Master y Doctorado. 2011 y 2014

\begin{tabular}{|l|c|c|c|c|c|c|c|c|}
\hline \multirow{2}{*}{} & \multicolumn{4}{|c|}{ Bases de cotización Máster } & \multicolumn{3}{c|}{ Bases de cotización Doctorado } \\
\cline { 2 - 10 } & \multicolumn{2}{|c|}{ Mujeres } & \multicolumn{2}{c|}{ Hombres } & \multicolumn{2}{c|}{ Mujeres } & \multicolumn{2}{c|}{ Hombres } \\
\cline { 2 - 9 } & 2011 & 2014 & 2011 & 2014 & 2011 & 2014 & 2011 & 2014 \\
\hline Media & 22220 & 23915 & 24806 & 27058 & 28486 & 31737 & 30171 & 33376 \\
\hline$\rho$-valor & \multicolumn{3}{|c|}{0,025} & \multicolumn{2}{|c|}{0,006} & 0,000 & 0,000 \\
\hline
\end{tabular}

Fuente: elaboración propia. Datos MECD.

\section{DISCUSIÓN Y CONCLUSIONES}

Este trabajo ha tenido como objetivo analizar las diferencias en los indicadores de inserción laboral de la tasa de afiliación, el trabajo acorde y la base de cotización en los niveles de posgrado de máster y doctorado entre los hombres y las mujeres y entre los años 2011 y 2014, a partir de los datos de inserción laboral del informe de Datos y Cifras del Sistema Universitario Español 2015-16 (MECD, 2016).

En primer lugar, se han presentado, de acuerdo con los datos del citado informe del Ministerio de Educación, los datos de las tasas de desempleo para el año 2014 que se sitúan tanto para máster como doctorado por encima de las de la OCDE y de la UE y entre las más elevadas entre los países analizados, en el caso del máster la segunda más alta, después de Grecia, y en el caso de doctorado la más alta. Estas tasas coinciden con un contexto de crisis económica, no obstante, merecen una profunda reflexión sobre las líneas de acción 
para ampliar la empleabilidad y facilitar los procesos de inserción profesional de nuestros egresados y nuestras egresadas.

Los resultados han puesto de manifiesto que las tasas de afiliación se incrementan entre los años 2011 y 2014 en el caso de los egresados y egresadas de máster, con un mayor incremento en las ramas de enseñanza de Ciencias Sociales y Jurídicas y Artes y Humanidades, mientras que disminuyen para el caso de doctorado, con un mayor descenso en Ingeniería y Arquitectura y Ciencias. Por ámbitos de estudio, la menor tasa de afiliación tanto en máster como en doctorado a los cuatro años de haber finalizado los estudios se da en Humanidades. En cuanto a la tasa de afiliación por rama de enseñanza y sexo para el caso de máster vemos que, tanto en 2011 como en 2014, es superior para los hombres en las ramas de Ciencias Sociales y Jurídicas e Ingeniería y Arquitectura, siendo superior para las mujeres en el resto de ramas de enseñanza, Artes y Humanidades, Ciencias de la Salud y Ciencias, mientras que, para el caso de doctorado en 2011, las mujeres tienen tasas de afiliación superiores en todas las ramas de enseñanza, a excepción de Ciencias de la Salud, y en 2014 dichas tasas son superiores para las mujeres en todas las ramas de enseñanza, dándose las mayores diferencias a favor de las mujeres en las ramas de Ciencias Sociales y Jurídicas y de Ciencias. Los resultados solo nos permiten confirmar parcialmente H1a, ya que las tasas de afiliación solo son significativamente superiores en los egresados de máster en 2011, mientras que en doctorado son superiores de manera significativa para las egresadas tanto en 2011 como 2014. A su vez, las tasas de afiliación de máster se incrementan de manera significativa entre 2011 y 2014, tanto en los hombres como en las mujeres, mientras que las tasas de afiliación de doctorado experimentan un descenso significativo entre 2011 y 2014 en el caso de los hombres, en consecuencia, solo podemos confirmar H1b en todos los casos para el nivel de máster.

En relación al trabajo acorde con el nivel formativo los resultados indican que, tanto para el año 2011 como 2014, los hombres tienen porcentajes superiores de trabajo acorde que las mujeres en todas las ramas de enseñanza en el caso del máster, mientras que para el caso del doctorado los porcentajes superiores de trabajo acorde de los hombres respecto de las mujeres, tanto al primer año de haber finalizado los estudios como al cuarto año, se dan en las ramas de enseñanza de Ingeniería y Arquitectura, Ciencias de la Salud y Ciencias. En el indicador de trabajo acorde en los estudios de máster hay diferencias significativas entre los hombres y las mujeres tanto en el 2011 como en el 2014, a favor de los hombres. En doctorado las diferencias significativas entre ambos sexos se dan también a favor de los hombres en 2014, por tanto, podemos confirmar H2a en todos los casos, salvo en el caso de los egresados y egresadas de doctorado en 2011 donde no se encuentran diferencias significativas entre hombres y mujeres. A su vez, podemos confirmar parcialmente H2b, ya que entre los años 2011 y 2014 se produce un descenso significativo del indicador de trabajo acorde al nivel formativo en las mujeres tanto en los estudios de máster como en doctorado, mientras que no se dan diferencias significativas en el caso de los hombres. 
Los resultados sobre el indicador de la base de cotización son concluyentes y nos permiten afirmar que la brecha de género está patente y opera en detrimento de las mujeres. En relación a las bases de cotización se observa que las mujeres en todos los niveles formativos, tanto en máster como en doctorado, en todos los momentos, tanto en el primer año de finalización de los estudios, 2011, como a los cuatro años, 2014, como en todas las ramas de enseñanza, las bases de cotización son inferiores a las de los hombres y, además, se incrementa la diferencia entre 2011 y 2014 de forma más acusada para el caso de máster que de doctorado. Así, las bases de cotización son significativamente superiores para los hombres, por lo que podemos confirmar H3a. Por tanto, en línea con los resultados explicitados por el Informe Español. Panorama de la Educación (OCDE, 2018) que indica que la brecha de género si bien opera a favor de las mujeres en el sistema educativo obteniendo mejores resultados académicos, lo hace en el sentido inverso y, por tanto, a favor de los hombres en el mercado laboral. En esta misma línea se pronuncia el informe Esenciales, $N .^{\circ} 39$ (BBVA-IVIE, 2019) en el caso de los egresados y egresadas de grado, para los que existen datos más recientes publicados por el SUE, que no incluye los datos de la formación de posgrado. Igualmente podemos confirmar $\mathrm{H} 3 \mathrm{~b}$, dado que se produce un incremento significativo de las bases de cotización entre el 2011 y 2014 tanto en hombres como mujeres y tanto en máster y como en doctorado.

A modo de conclusión general podríamos señalar que si bien, a la vista de los resultados, no se observan grandes diferencias de género en cuanto a la tasa de afiliación, incluso siendo en muchos casos más favorable para las mujeres, a medida que se analizan indicadores que podríamos considerar más relacionados con la calidad del empleo, como el trabajo acorde con el nivel formativo y la base de cotización, se va incrementando la brecha de género en detrimento de las mujeres. Si bien, multitud de factores pueden dar lugar a estos resultados, resulta obvio que sigue siendo un reto impulsar políticas públicas para favorecer la igualdad de oportunidades de los egresados y egresadas de la formación de posgrado en el mercado laboral.

\section{REFERENCIAS}

BBVA-IVIE (2019). Inserción laboral de los universitarios (Esenciales, n. ${ }^{\circ}$ 39). Bilbao: Fundación BBVA. Recuperado de: https://www.fbbva.es/esencial/

Bravo Salinas, N. H. (2007). Competencias Proyecto Tuning-Europa, Tuning-Amèrica Latina. Recuperado de: http://www.cca.org.mx/profesores/cursos/hmfbcp_ut/pdfs/m1/ competencias_proyectotuning.pdf

Comisión Europea/EACEA/Eurydice (2015). El Espacio Europeo de Educación Superior en 2015: Informe sobre la implantación del Proceso de Bolonia. Luxemburgo: Oficina de Publicaciones de la Unión Europea. Recuperado de: http://publications.europa.eu/ resource/cellar/91f926b2-6965-4abe-a1be-600903e4df93.0003.01/DOC_1 
Conferencia de Ministros Europeos Responsables de Educación Superior (2001). Towards the European Higher Education Area. Communiqué of the meeting of European Ministers in charge or Higher Education en Prague on May 19th 2001. Recuperado de: http://www.ehea.info/media.ehea.info/file/2001_Prague/47/4/2001_Prague_ Communique_Spanish_553474.pdf

Conferencia de Ministros Europeos Responsables de Educación Superior (2007). Towards the European Higher Education Area: Responding to Challenges in a Globalised World. London, 18 May. Recuperado de: http://www.ehea.info/media.ehea.info/ file/2007_London/70/4/2007_London_Communique_Spanish_588704.pdf

Conferencia de Ministros Europeos Responsables de Educación Superior (2012). Making the Most of Our Potential: Consolidating the European Higher Education Area. Communique of the Conference of Ministers responsible for Higher Education in Bucharest on April 26-27th 2012. Recuperado de: http://www.ehea.info/media.ehea.info/ file/2012_Bucharest/67/3/Bucharest_Communique_2012_610673.pdf

Conferencia de Ministros Europeos Responsables de Educación Superior (2018). Paris Communiqué. Communique of the Conference of Ministers responsible for Higher Education in Paris on May 25th 2018. Recuperado de: http://www.ehea.info/media.ehea. info/file/2018_Paris/77/1/EHEAParis2018_Communique_final_952771.pdf

Declaración Conjunta de los Ministros de Francia, Alemania, Italia y el Reino Unido (1998). Declaración conjunta para la armonización del diseño del Sistema de Educación Superior Europeo de 25 de mayo de 1998: Declaración de la Sorbona. Recuperado de: http:// www.eees.ua.es/documentos/declaracion_sorbona.htm

Declaración Conjunta de los Ministros Europeos de Educación (1999). El Espacio Europeo de Enseñanza Superior. Declaración conjunta de los Ministros Europeos de Educación reunidos en Bolonia el 19 de junio de 1999: Declaración de Bolonia. Recuperado de: http://www.ehea.info/media.ehea.info/file/Ministerial_conferences/06/0/1999_ Bologna_Declaration_Spanish_553060.pdf

Echeverria, B. \& Martínez, P. (2019). Revolución 4.0, Competencias, Educación y Orientación. Revista Digital de Investigación en Docencia Universitaria, 12 (2). DOI: https:// doi.org/10.19083/ridu.2018.831

González, J. \& Wagenaar, R. (2003). Tuning Educational Structures in Europe. Informe final. Bilbao: Universidad de Deusto.

Ley Orgánica 4/2007, de 12 de abril, por la que se modifica la Ley Orgánica 6/2001, de 21 de diciembre, de Universidades. Boletín Oficial del Estado, 13 de abril de 2007, núm. 89, pp. 16241-16260. 
Ministerio de Ciencia, Innovación y Universidades (2019). Inserción Laboral de los Egresados Universitarios. Curso 2013-2014 (análisis hasta 2018). Madrid: MCIU. Recuperado de: http://www.ciencia.gob.es/stfls/MICINN/Universidades/Ficheros/Estadisticas/INFORME_INSERCION_2013_14.pdf

Ministerio de Educación, Cultura y Deporte. (2013). Objetivos educativos europeos y españoles. Estrategia Educación y Formación 2020. Informe español 2013. Madrid: MECD. Recuperado de: https://www.educacionyfp.gob.es/inee/dam/jcr:5b7ee19b-7981-445b886a-50070ba74ac3/objetivos20202013.pdf

Ministerio de Educación, Cultura y Deporte (2015). Inserción laboral de los egresados universitarios. La perspectiva de la afiliación a la Seguridad Social. Madrid: MECD. Recuperado de: https://www.educacionyfp.gob.es/dam/jcr:3469cbcd-0dfa-459e-a5c9f27285b72121/insercion-laboral-egresados-universitarios-.pdf

Ministerio de Educación, Cultura y Deporte. (2016). Datos y cifras del sistema universitario español. Curso 2015-2016. Madrid: MECD. Recuperado de: http://www.educacionyfp.gob.es/dam/jcr:6a538b72-1866-4100-b1b7-b3fd26bb8191/datos-y-cifras-sue2015-16-web-.pdf

Ministerio de Educación, Cultura y Deporte (2018). Panorama de la Educación. Indicadores de la OCDE 2018. Madrid: MECD. Recuperado de: http://www.educacionyfp.gob. es/inee/dam/jcr:2cba4aaa-4892-40d7-ac8b-00efbc95b8a2/Panorama\%20de\%20la\%20 Educacion\%202018_final.pdf

Real Decreto 1393/2007, de 29 de octubre, por el que se establece la ordenación de las enseñanzas universitarias oficiales. Boletín Oficial del Estado. Madrid, 30 de octubre de 2007, núm. 260, pp. 44037-44048.

Real Decreto 861/2010, de 2 de julio, por el que se modifica el Real Decreto 1393/2007, de 29 de octubre, por el que se establece la ordenación de las enseñanzas universitarias oficiales. Boletín Oficial del Estado. Madrid, 3 de julio de 2010, núm. 161, pp. 58454-58468.

Real Decreto 99/2011, de 28 de enero, por el que se regulan las enseñanzas oficiales de Doctorado. Boletín Oficial del Estado. Madrid, 10 de febrero de 2011, núm. 35, pp. 13909-13926.

Real Decreto 1027/2011, de 15 de julio, por el que se establece el Marco Español de Cualificaciones para la Educación Superior. Boletín Oficial del Estado. Madrid, 3 de agosto de 2011, núm. 185, pp. 87912-87918.

Real Decreto 43/2015, de 2 de febrero, por el que se modifica el Real Decreto 1393/2007, de 29 de octubre, por el que se establece la ordenación de las enseñanzas universita- 
rias oficiales, y el Real Decreto 99/2011, de 28 de enero, por el que se regulan las enseñanzas oficiales de doctorado. Boletín Oficial del Estado. Madrid, 3 de febrero de 2015, núm. 29, pp. 8088-8091.

Real Decreto 195/2016, de 13 de mayo, por el que se establecen los requisitos para la expedición del Suplemento Europeo al Título Universitario de Doctor. Boletín Oficial del Estado. Madrid, 3 de junio de 2016, núm. 134, pp. 36611-36628.

Rodríguez, S., Prades, A., Bernáldez, L. \& Castiñeira, S. (2010). Sobre la empleabilidad de los graduados universitarios en Catalunya: del diagnóstico a la acción. Revista de Educación, 351, 107-137. 